\title{
Improving the efficacy of elderly patients' hospital discharge through multi- professional safety briefings and behavioural change
}

\author{
Joanna Corrado, Kathryn Topley, Alison Cracknell
}

Leeds Teaching Hospitals NHS Trust, UK

\begin{abstract}
At Leeds Teaching Hospitals NHS Trust doctors type an electronic discharge advice note (eDAN) which includes a prescription for discharge medication, before a patient can be discharged from hospital. In 2014 staff on the Medical Admissions Unit for Older People identified significant delays in the completion of this document, with an average completion time of 138 minutes. This caused patient harm and exacerbated bed management problems as patients remained in hospital longer than necessary to obtain their discharge medication. Ward staff wanted to improve the efficacy of older peoples' discharge by speeding up this process in as safe a manner as possible. A number of interventions were tested, led by junior doctors in a 'bottom-up' leadership strategy. Interventions included a daily discharge briefing to recap discharges and help junior doctors prioritise workload. After several months of sustained effort, the average time to complete eDANs fell by over an hour resulting in discharge medication dispensed earlier in the day and improved patient safety.
\end{abstract}

\section{Problem}

Before patients are discharged from hospital, junior doctors are required to complete a discharge document which includes a prescription of discharge medication and a summary of the hospital admission for the benefit of the general practitioner. At Leeds Teaching Hospitals NHS Trust (LTHT) in West Yorkshire, this document is completed electronically and is known as an electronic discharge advice note (eDAN). These are typed by doctors once it is known that a patient is ready for discharge and the discharge prescription is then processed by the hospital pharmacy before this medication is sent back to the ward for the patient.

Staff on the Medical Admissions Unit for Older People at LTHT had identified that there was a considerable delay in doctors completing eDANs with a consequent delay in discharge medication reaching the ward. This resulted in patient harm in two ways; some patients chose to leave hospital rather than wait for their discharge medication, resulting in missed medication doses. Others remained in hospital longer than was necessary for their medication, taking up beds which could be used for other patients and exacerbating problems with hospital capacity particularly over the winter months.

Staff on the Medical Admissions Unit for Older People at LTHT wanted to improve the discharge process by speeding up eDAN completion

\section{Background}

Timely eDAN completion is important not only to facilitate the discharge process from hospital, but also to allow rapid communication from hospital to primary care, improving continuity of care and patient safety $[1,2]$. Nevertheless, its prioritisation can be challenging when junior doctors have a high workload or staffing levels are low. Completing eDANs promptly is more challenging to sustain in an acute hospital environment such as a Medical Admissions Unit due to the nature of the ward; there is often a very high turnover of patients, a significant proportion of whom may be acutely unwell. A similar challenge was described in a recent quality improvement project on the ICU at Kings College Hospital [3]. On the Medical Admissions Unit for Older People at LTHT there are approximately 20-50 discharges each week and also a new team of junior doctors rotating through weekly. Staff therefore wanted to produce sustainable interventions to reduce delays in the preparation of discharge medications while still delivering a high standard of patient care on the ward.

The study was multi-professional but led by junior doctors in conjunction with consultants, nurses, and pharmacists. The behavioural change methods were supported by the Yorkshire and Humber Improvement Academy.

\section{Baseline measurement}

Baseline data was collected between April and May 2014. The data collected included time taken for:

- doctors to commence and complete an eDAN once a discharge decision was made

- pharmacy department to release medication to the ward

This showed considerable variability in time taken for doctors to complete the eDAN before sending it on to pharmacy, ranging from 10 to 370 minutes (average 138 minutes).

\section{Design}

Staff felt that the most successful intervention would be one which helped junior doctors prioritise their workload effectively so that eDANs could be completed promptly once patients were known to 
BMJ Quality Improvement Reports

be ready for discharge. The first intervention tested therefore, was a discharge briefing integrated into the daily multi-disciplinary team (MDT) meeting highlighting patients for:

- imminent discharge

- discharge later that day pending investigations

- expected discharge within 24 to 48 hours

This simple but effective approach helped junior doctors and pharmacists prioritise eDANs appropriately and allocate responsibility for their completion.

Other interventions which were tested included making changes to the doctors' handover document to include a section on discharge, drafting extra help in from other wards when discharge rates were particularly high, completing eDANs during the MDT meeting in order to use time as productively as possible and also allocating one member of the ward team to eDAN completion for that day.

Suggestions for interventions came mainly from junior doctors. These were discussed with the consultants in a 'bottom-up' leadership strategy and then trialled using PDSA methodology. As interventions were tested all MDT members became involved with the project. The project's progress was discussed at weekly meetings supported by the Improvement Academy.

\section{Strategy}

PDSA cycle 1 June - July 2014: discharge briefings were tested three times a week during the MDT meeting. Doctors also made changes to their handover document to include a section on discharge. Results of data collection at this time did not show a significant improvement in time taken for doctors to complete eDANs. However, the consensus of ward staff was that the briefings were helping doctors to prioritise eDAN completion and as a result the discharge process seemed to be running more smoothly. We therefore decided to continue with this intervention.

PDSA cycle 2 July - September 2014: following positive feedback from staff the discharge briefings were increased to 5 times a week. Other interventions were drafting in junior doctors from other wards to help complete eDANs on days when the discharge rate was particularly high or if staffing levels were low. We also started to allocate one team member each day to eDAN completion.

PDSA cycle 3 - September 2014 onwards: the discharge briefings had become an established part of the ward routine, and we noticed improvement in data collected; eDAN completion was significantly quicker than previously. More encouragingly, the general discharge process on the ward was more efficient and many eDANs were completed in advance meaning that discharge medication was already on the ward once a patient had been deemed ready for discharge. We also tested another intervention during this time; assigning one team member to complete eDANs during the MDT meeting. However we found that this was not successful as often this team member missed out on vital information during the meeting. This was therefore not continued.

\section{Results}

Data was collected weekly throughout the project to determine the time taken for doctors to complete an eDAN and the subsequent pharmacy turnaround time. This was analysed by the Improvement Academy and SPC charts produced with annotations for when interventions were implemented. These were shared with ward staff at the daily briefing to demonstrate the impact of the ward efforts.

Our data show that over time the average time for doctors to produce the eDAN fell by 63 minutes. This improvement has been sustained over several months. There is also considerably less variability in the time taken to complete an eDAN and many eDANS are produced in advance of a patient's discharge meaning that medication is ready on the ward as soon as a decision has been made that a patient can be discharged (Figure 1).

See supplementary file: ds5788.jpg - "Figure 1"

\section{Lessons and limitations}

Our intervention ideas were all very simple which made their implementation easier and the ideas originated from junior team members with the most experience in eDAN preparation.

This project reinforced that sustained improvement is a team effort; members need to understand the problem, suggest interventions and see impact in a timely manner. Sharing the results of the team's efforts in the form of run charts served as a visual representation of the impact of interventions and was useful in generating new ideas. In future we would involve the wider team in data collection rather than assigning only one team member to this role.

\section{Conclusion}

The preparation of eDANs can often seem like a low priority task for junior doctors but it is one which can have a significant impact on patient safety. These interventions are now being tested on other wards throughout the Trust in order to try to improve timely patient discharge.

Our interventions were ultimately successful but it took several months before a sustained improvement was noted. This demonstrates the need to persevere with quality improvement projects, learn from unsuccessful interventions and refine them if necessary in order to bring about sustained improvement.

\section{References}

1. Barr R, Chin KY, Yeong K. Improving transmission rates of electronic discharge summaries to GPs. BMJ Quality Improvement Reports. 2013;(2):1

2. Moore C, Wisnivesky J, Williams S, McGinn T. Medical errors related to discontinuity of care from an inpatient to an outpatient setting. Journal of General Internal Medicine. 
2003;18(8):646-51.

3. Goulding L, Parke H, Maharaj R et al. Improving critical care discharge summaries: a collaborative quality improvement project using PDSA. BMJ Quality Improvement Reports. 2015;(4):1

\section{Declaration of interests}

Nothing to declare

\section{Acknowledgements}

Many thanks to:

The Yorkshire and Humber Improvement Academy for supporting this work, particularly Michael Rooney for data analysis and creation of run charts.

All staff on ward 27 at St James's University Hospital for continued efforts in supporting quality improvement projects. Particular thanks to:

Janet Darlington

Heather Smith

\section{Ethical approval}

According to the policies regarding research at Leeds Teaching Hospitals NHS Trust this project was felt to be exempt from ethics review. 